\title{
Determinantes sociales en salud: su relación con el síndrome metabólico
}

\author{
Abigail Fernández Sánchez,* Sandra Hernández Corral,** María Guadalupe Ojeda Vargas***
}

\begin{abstract}
RESUMEN
El propósito de esta investigación es hacer una revisión del síndrome metabólico, su impacto en la salud de la población adulta y la intervención de los servicios de salud por medio de programas, así como su abordaje en los ámbitos biológico, político, económico y social, llamados determinantes sociales. El perfil epidemiológico, demográfico y económico conlleva la salud o enfermedad de la población. En el mundo 1,600 millones de personas tienen sobrepeso; de ellas, 400 millones son obesas. Los índices de morbimortalidad prematura se han incrementado. Las complicaciones por enfermedad cerebrovascular y la diabetes afectan la calidad de vida de la población y los presupuestos sanitarios. En México hay más de 17 millones de hipertensos, 14 millones de dislipidémicos, seis millones de diabéticos y más de 35 millones de adultos -siete de cada diez- con sobrepeso u obesidad. Presentamos un recorrido histórico del proceso-establecimiento del síndrome metabólico. En la actualidad las organizaciones American Heart Association/National Heart, Lung and Blood Institute y el Panel de Tratamiento del Colesterol han establecido los criterios para definir esa patología. Se describen y analizan los determinantes sociales como un entramado de factores estructurales e intermediarios políticos, ambientales y sociales que acotan el estado de salud de los individuos y las comunidades. Es necesario conjuntar las estrategias de los sistemas de salud, políticas públicas y el colectivo de la población para mejorar la salud.
\end{abstract}

Palabras clave: Determinantes sociales, síndrome metabólico.

\section{Social Determinants of Health: its relationship with the metabolic syndrome}

\begin{abstract}
The purpose of this research is to review metabolic syndrome, its impact on the health of adult population, the intervention of health services through programs, as well as its approach in the biological, economic, political and social health areas, the so-called social determinants. The epidemiological, demographic and economic profile of a population leads to its health or illness. 1.6 million people worldwide are overweight, of which 400 million are obese. The rate of morbidity and premature mortality has increased. Complications from cerebrovascular disease and diabetes affect the quality of life of the population and the health budgets. In Mexico, more than 17 million people are hypertensive, 14 million dyslipidemic, 6 million diabetic, and more than 35 million adults -seven out of every ten-are overweight or obese. We present a historical view of metabolic syndrome. Today organizations such as the American Heart Association/National Heart, Lung and Blood Institute and the Cholesterol Treatment Panel have established criteria for defining such pathology. We analyze and describe the social determinants as a set of structural factors and political, environmental and social intermediaries that strongly influence the health status of individuals and communities. It is necessary to aggregate the strategies of the health systems, public policies and the general population to attain an overall health improvement.
\end{abstract}

Key words: Social determinants, metabolic syndrome.

* Universidad Autónoma del Estado de Morelos.

** Instituto Nacional de Rehabilitación.

*** Universidad de Guanajuato.

Correspondencia: Abigail Fernández Sánchez. Privada del Hule Núm. 7, Col: Granjas. Cuernavaca, Morelos. Tel: 01-777-2241583.

E-mail: abifer2002@yahoo.com

Este artículo puede ser consultado en versión completa en http://www.medigraphic.com/enfermerianeurologica 


\section{INTRODUCCIÓN}

E síndrome metabólico es un problema de salud pública del siglo XXI en todo el mundo. Se experimenta con gran preponderancia en la vida adulta; por lo tanto, cada cuatro segundos ocurre un infarto agudo del miocardio y cada cinco segundos un evento vascular cerebral. La OMS indica que en el 2005 existían en el mundo aproximadamente 1,600 millones de personas con sobrepeso, de las cuales 400 millones eran obesas, y proyecta que para el 2015 habrá alrededor de 2,300 millones de personas con sobrepeso y 700 millones de obesas. ${ }^{1} \mathrm{La}$ enfermedad cerebrovascular y la diabetes afectan la calidad de vida de la población y los presupuestos sanitarios de muchos países desarrollados o en vías de desarrollo. En América Latina 75\%, asociado cinco veces en la prevalencia de la diabetes tipo 2 y de 2 a 3 veces más en la enfermedad cerebrovascular. $^{2}$

En México, entre la población adulta de 20 a 69 años, hay más de 17 millones de hipertensos (30\%), 14 millones de dislipidémicos (28\%), seis millones de diabéticos y más de 35 millones con sobrepeso u obesidad (43\%). Como resultado, el $8 \%$ del total de la población -que equivale a siete de cada diez personas adultas- presenta sobrepeso u obesidad. Otro dato relevante es que cerca del $70 \%$ de los mexicanos que viven en los Estados Unidos padecen sobrepeso u obesidad, tanto hombres como mujeres..$^{2-3}$

El acelerado proceso de urbanización de los países latinoamericanos y los estilos de vida adoptados de otros países de mayor desarrollo dieron origen a un aumento en la prevalencia del síndrome.

\section{ANTECEDENTES}

El síndrome metabólico es definido para su diagnóstico por organizaciones como la Organización Mundial de la Salud (OMS), el Panel de Tratamiento del Colesterol en Adultos en su tercera versión (ATPIII) y la Federación Internacional de Diabetes (FID) (Cuadros I y II) como un "conjunto de alteraciones metabólicas constituido por la obesidad de distribución central, la disminución de las concentraciones del colesterol unido a las lipoproteínas de alta densidad

Cuadro I. Revisión histórica de la denominación de síndrome metabólico.

\begin{tabular}{|c|c|c|}
\hline Año & Autor & Hallazgos \\
\hline 1923 & Kylin, médico sueco ${ }^{4}$ & $\begin{array}{l}\text { Refiere las primeras descripciones de la asociación entre diabetes mellitus, hipertensión } \\
\text { arterial e hiperuricemia }\end{array}$ \\
\hline 1927 & Marañon ${ }^{4}$ & \\
\hline 1947 & Vague $^{4}$ & \\
\hline 1960 & Crepaldi & \\
\hline 1967 & Avogaro SM & \\
\hline 1988 & & En la conferencia de Banting \\
\hline 1999 & Gerald M Reaven 5 & Síndrome "X" \\
\hline 2001 & Organización Mundial de la Salud & Primera definición oficial de síndrome metabólico \\
\hline 2002 & ES Ford & Tercer reporte del National Cholesterol Education Program (NCEP) \\
\hline 2005 & R Khan & $\begin{array}{l}\text { Prevalencia del síndrome metabólico en Estados Unidos, frecuencia inversa de documento } \\
\text { (inverse document frequency, IDF), Consenso Mundial del Panel de Tratamiento del Colestero } \\
\text { (ATPIII) }\end{array}$ \\
\hline 2006 & $\begin{array}{l}\text { Nueva iniciativa de la Asociación } \\
\text { Norteamericana de Diabetes (ADA) }\end{array}$ & Cuidado de la diabetes \\
\hline 2006 & Scott y Grundy (mencionado por Pérez) ${ }^{6}$ & $\begin{array}{l}\text { Prediabetes, síndromes plurimetabólico y cardiometabólico, hipertensión, dislipidemia, circun- } \\
\text { ferencia de cintura, hipertrigliceridemia }\end{array}$ \\
\hline 2007 & Roberto R. Pérez Moreno ${ }^{6}$ & $\begin{array}{l}\text { El cuarteto de la muerte, síndrome de insulino-resistencia, dislipemia aterogénica, síndrome } \\
\text { dismetabólico }\end{array}$ \\
\hline 2008 & Sánchez, Jaramillo y colaboradores ${ }^{7}$ & $\begin{array}{l}\text { Compuesto por elementos de sustrato común a la resistencia a la insulina, es factor de riesgo } \\
\text { para desarrollar enfermedad cardiovascular y diabetes }\end{array}$ \\
\hline
\end{tabular}

Fuente: González, Bautista, Irigoyen (2009). ${ }^{8}$

Cuadro II. Integración del síndrome metabólico por diversas organizaciones. ${ }^{5,9}$

Organización Mundial de la Salud, 1999. American Heart Association/National Heart, Lung and Blood Institute (AHA/NHLBI), 2005

Panel de Tratamiento del Colesterol en Adultos, en su tercera versión (NCEP ATPIII), 2001

Federación Internacional de Diabetes (FID), 2005

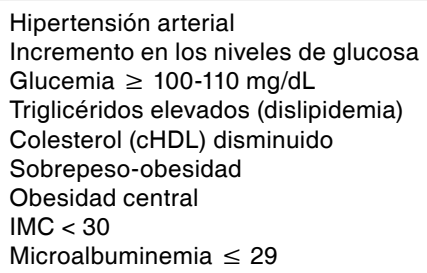


(cHDL), la elevación de las concentraciones de triglicéridos, el aumento de la presión arterial y la hiperglucemia". ${ }^{4}$

La obesidad y/o los antecedentes genéticos culminan en diabetes mellitus e hipertensión arterial, induciendo en su trayecto un compromiso cardiovascular y metabólico relacionado. El asociar las alteraciones lipídicas a la diabetes y la obesidad incrementa el riesgo cardiovascular. Se ha encontrado un patrón común en la patogénesis de estas enfermedades (Cuadro II). Algunos factores sociales y demográficos como la raza, sexo, edad, situación geográfica y economía influyen en los índices de obesidad que han generado el aumento del número de personas con síndrome metabólico en las regiones en desarrollo. ${ }^{9}$ Otros factores que contribuyen a su aparición son el sedentarismo, los estilos de vida, la transición alimentaria, la baja cobertura del primer nivel de atención, y la pobreza, entre otros. Esto hace que cada día se incremente el número de casos en la población. Es importante identificar los determinantes sociales que conllevan a su aparición para poder prevenirlos, desde el punto de vista físico, psicológico, social y económico, puesto que persiste en un círculo vicioso: pobreza-enfermedadpobreza, potenciado por la desigualdad de oportunidades de los individuos. ${ }^{10}$

Las instituciones afirman establecer en sus programas estrategias de control de los riesgos y capacitación del personal de salud, además de la creación de nuevas políticas para la atención de las personas afectadas, cuyo número crecerá aun más de manera importante en las décadas por venir. ${ }^{11}$ El síndrome metabólico se estudia con mediciones bioquímicas de triglicéridos, colesterol y glucosa. Sin embargo, es importante tener en cuenta la complejidad del síndrome, resultado de la interrelación de factores ambientales, culturales, sociales y económicos (determinantes estructurales intermediarios), y no conformarse con el establecimiento de un diagnóstico meramente bioquímico.

\section{DESARROLLO}

La OMS declaró que existe una prevalencia del síndrome metabólico en el 33.6\% de la población adulta. En España, la prevalencia es del 24\%; ${ }^{12}$ en Singapur, del $30 \% ;{ }^{12}$ en la población americana, del 34.6 y $39.1 \%$, de acuerdo con los criterios del ATPIII y la FID, respectivamente, ${ }^{13}$ aunque con grandes variaciones entre un país y otro: en Canadá, del $19.1 \%:{ }^{10}$ en los Estados Unidos fue del $21.8 \%$ en el 2002:12 en Chile, del 37\%;12 en Colombia, con criterios del ATPIII, del $25.4 \%$ y con criterios de la FID, del $31.5 \%$; ${ }^{14}$ en Uruguay, del $27.7 \%{ }^{15}$ y en México del 46.5, 43.3 y $36.5 \%$ con criterios del ATPIII, la FID y la OMS, respectivamente. ${ }^{16}$ Como se puede apreciar, se han encontrado prevalencias muy distintas entre un país y otro y en el mismo país, sin dejar de observar que, independientemente del criterio de medición utilizado y el número de población, el problema existe y causa estragos. Algunos países son más afectados que otros, como es el caso de Latinoamérica y Estados Unidos, cuya prevalencia es mayor en relación con países como Francia y Singapur.

Es importante considerar los años perdidos a causa del síndrome metabólico, por una morbimortalidad prematura. La disminución de la expectativa de vida va en relación con el incremento de la mortalidad. En la población de mujeres con diabetes, su expectativa de vida disminuye en un $6.3 \%$, y en aquellas con enfermedades isquémicas del corazón, en un $2.0 \%$; en los hombres, las enfermedades isquémicas del corazón disminuyen la expectativa de vida en un $3.5 \%$ y la diabetes, en un $4.5 \%$. En las mujeres la diabetes ocupa el segundo lugar y las enfermedades isquémicas del corazón el quinto, tanto en poblaciones urbanas como en las rurales.17 A nivel mundial, México ocupa el segundo lugar en obesidad y el noveno lugar en diabetes mellitus. En cuanto a la distribución por sexo, en mujeres: diabetes mellitus $16.3 \%$, enfermedades isquémicas del corazón $10.6 \%$ y enfermedad cerebrovascular $6.5 \%$. En hombres: diabetes mellitus $11.3 \%$, enfermedades isquémicas del corazón $10.9 \%$, cirrosis y otras enfermedades crónicas del hígado $7.6 \%$ y enfermedad cerebro-vascular $4.7 \%(2005) .^{18}$

\section{BREVE RESEÑA HISTÓRICA}

En 1946 la OMS definió salud como "el completo bienestar físico, mental y social". Desde esta perspectiva se busca cubrir el bienestar en el contexto social con un enfoque biomédico. Aunque esta visión se ha tratado de mantener, no se ha satisfecho plenamente, por el contexto económico y político. En los años 60 y 70, en Latinoamérica, se integran programas de salud comunitaria con un enfoque parcial del cuidado de la salud, debido a la menor posibilidad de uso de las tecnologías, por su alto costo.

En relación con los determinantes sociales, Canadá es un país pionero. El ministro de salud Marc Lalonde ha expresado que, en cuanto a condicionantes de la salud, la biología humana ocupa un $20 \%$, el medio ambiente $20 \%$, los estilos de vida $50 \%$ y la organización de los servicios de salud $10 \% .{ }^{19}$ En el entorno de México, la inversión es dirigida hacia los servicios médicos, en tanto que los estilos de vida reciben una proporción mínima; por lo tanto, el impacto en la salud es reducido. ${ }^{21}$

En América Latina la comprensión de la salud desde un proceso que trasciende la simple causalidad se ha denominado 'determinación social'. Breihl ${ }^{21}$ y Castellanos ${ }^{22}$ explican que la salud no solo depende de los servicios de salud ni de las características biológicas humanas, sino que también está fuertemente influida por los modos, condiciones y estilos 
de vida que las sociedades imponen a las poblaciones. Este enfoque no fue entonces adoptado por la OMS.

En 1978 se lleva a cabo la conferencia de Alma-Ata "Salud para todos en el año 2000". El punto de partida de la OMS con relación a los determinantes sociales son los medioambientes en los que se desarrolla el individuo, la familia y la comunidad. El mensaje va acompañado de esperanza para un mejoramiento de las condiciones de salud de las poblaciones. Por otra parte, el discurso economicista, con fuertes debates y controversias encaminados a reformar los sistemas de salud, conduce a prácticas particulares. Los marcos interpretativos producen distintas miradas sobre la producción en salud y la desigualdad social (salud y educación), de manera que se eliminan las dimensiones sociales y solo se priorizan los problemas de salud emergentes, lo cual deviene en un detrimento en las condiciones de vida y salud de las poblaciones..$^{20,26}$

Más tarde emerge la epidemiología como brazo "diagnóstico" de la salud colectiva y sufre las tensiones, impulsos y obstáculos de todo conocimiento que contribuye a definir la imagen de la realidad, y el éxito o fracaso de la política. La información epidemiológica se torna cada vez más un instrumento socialmente valorado. Esa circunstancia histórica nos lleva a sostener, desde los comienzos de la era neoliberal, que la epidemiología se construye "entre fuegos". ${ }^{21}$
Se pueden definir los determinantes sociales como un "conjunto de factores personales, sociales, económicos y ambientales que determinan el estado de salud de los individuos o poblaciones". Entre estos factores se incluyen conductas, estilos de vida saludables, ingresos y posición social, educación, trabajo y condiciones laborales, el acceso a los servicios sanitarios adecuados y los entornos físicos. ${ }^{20,23}$

El reconocimiento de la naturaleza social de la salud no es un asunto nuevo en la historia de la salud pública, puesto que ha logrado un importante desarrollo durante los siglos XIX y XX. El XXI es testigo del llamado emergente para incorporar esta perspectiva en la investigación y el desarrollo de políticas sociales y económicas que condicionen el crecimiento, la vida y el trabajo. ${ }^{23}$

En cuanto a la situación social en la Unión Europea y América Latina, en los últimos años se realizan esfuerzos para dar impulso a programas y proyectos para enfrentar la pobreza, la desigualdad y la exclusión, con el fin de contribuir a la cohesión social que orienta las políticas sociales promovidas por la cooperación descentralizada. Así mismo, es importante impulsar proyectos, programas sociales de ámbito local, la participación, una nueva gobernanza local y la promoción de la participación ciudadana. ${ }^{24}$

Cuadro III. Modelos de determinantes sociales

\begin{tabular}{|c|c|c|}
\hline Modelo & Contexto & Categorías \\
\hline $\begin{array}{l}\text { Esquema general de la producción } \\
\text { de inequidades en salud, propues- } \\
\text { to por el equipo de equidad de la } \\
\text { Organización Mundial de la Salud }\end{array}$ & $\begin{array}{l}\text { Sociopolítico: } \\
\text { - Posición socioeconómica } \\
\text { - Determinantes sociales o estructurales de las inequida- } \\
\text { des sanitarias } \\
\text { Ej. estratificación social mediante la educación, el mercado } \\
\text { laboral y la cohesión social } \\
\text { - Desigualdades sociales }\end{array}$ & $\begin{array}{l}\text { - Estado de salud diferencial } \\
\text { - Sistema sanitario } \\
\text { Determinantes específicos o posición social de una } \\
\text { persona } \\
\text { Vía o determinantes intermediarios o sociales y de salud } \\
\text { Vulnerabilidad, exposición diferenciada } \\
\text { Ej. Condiciones de vida y de trabajo. Comportamientos } \\
\text { saludables, atención médica y social en el transcurso } \\
\text { de la vida }\end{array}$ \\
\hline $\begin{array}{l}\text { Dahigren y Whitehead, producción } \\
\text { de inequidad en la salud }\end{array}$ & $\begin{array}{l}\text { - Social } \\
\text { - Políticas }\end{array}$ & $\begin{array}{l}\text { - Posición social } \\
\text { - Exposición y vulnerabilidad diferencial } \\
\text { - Enfermedades o lesiones } \\
\text { - Consecuencias sociales de la mala salud }\end{array}$ \\
\hline $\begin{array}{l}\text { Castellanos, procesos determi- } \\
\text { nantes, niveles en salud pública }\end{array}$ & $\begin{array}{l}\text { - Modos de vida } \\
\text { - Condiciones de vida }\end{array}$ & $\begin{array}{l}\text { - Individual } \\
\text { - Social } \\
\text { - Grupos de población }\end{array}$ \\
\hline Wilkinson y Marmot, 2003 & $\begin{array}{l}\text { Estructura y entorno social } \\
\text { - Psicológicos } \\
\text { - Comportamiento de la salud }\end{array}$ & $\begin{array}{l}\text { - Factores materiales } \\
\text { - Salud: mental y fisiológica desde la niñez, genes y } \\
\text { cultura } \\
\text { - Bienestar, morbilidad y mortalidad }\end{array}$ \\
\hline Modelo de sistemas de Bonferrer & $\begin{array}{l}\text { - } \text { Microsistema } \\
\text { - Mesosistema } \\
\text { - Macrosistema }\end{array}$ & \\
\hline $\begin{array}{l}\text { Determinantes estructurales e } \\
\text { intermediarios, de Breilh }{ }^{24}\end{array}$ & $\begin{array}{l}\text { - Determinantes estructurales } \\
\text { - Determinantes intermediarios } \\
\text { (Categorizados en circunstancias materiales-personales) }\end{array}$ & $\begin{array}{l}\text { - Posición socioeconómica } \\
\text { - Estructura social } \\
\text { - Elase social: relaciones de género y etnia } \\
\text { - } \text { alimentoción, ocupación, ingreso, disponibilidad de } \\
\text { - Comportamientos, factores biológicos y psicosocia- } \\
\text { - Sistema de salud } \\
\text { - Estructura social y condiciones de vida y trabajo }\end{array}$ \\
\hline
\end{tabular}


Desde la mirada de la medicina social, los determinantes sociales en salud conllevan a la construcción de pensamiento con un enfoque particular en salud, en el contexto regional. Breihl ${ }^{24}$ expone cómo la determinación social de la salud se interrelaciona con el modo y los estilos de vida y con los perfiles de los problemas de salud, en lo que denomina la dialéctica entre lo singular (individuo), lo particular (grupos) y lo general (las poblaciones).

\section{MODELOS CONCEPTUALES DE LOS DETERMINANTES SOCIALES ESTRUCTURALES E INTERMEDIARIOS EN LA SALUD}

Los modelos de los determinantes sociales (Cuadro III) muestran la determinación social, el impacto y la equidad en salud. Reflejan desde una relación de la salud individualcolectiva, estilos de vida individual relacionados con las redes sociales y comunitarias; a su vez todas éstas se vinculan con el ambiente, producción, educación, condiciones de vida y trabajo, servicios (sanitarios, agua), vivienda y atención a la salud. Por último, confluyen las condiciones socioeconómicas, culturales y medioambientales. ${ }^{23}$

Según el modelo de Breilh, los determinantes estructurales e intermediarios están constituidos en el contexto sociopolítico: tipo de gobierno, políticas económicas, sociales y públicas, así como la cultura y los valores sociales.

En cuanto al gradiente social, se encuentra intrínsecamente vinculado a las inequidades en salud, que son una razón de peso subyacente a las grandes diferencias que presenta en el mundo la esperanza de vida. Permite comprender por qué las personas pobres y marginadas enferman más y mueren antes que las personas que gozan de mejor posición social. En México, la posición socioeconómica y la estructura social ha sido afectada por las desigualdades dadas por un contexto históricosocial. Esto incluye la clase social, las relaciones de género y la etnia. Las condiciones de salud de la población indígena se ven afectadas por los factores económicos y las condiciones institucionales; reflejan tanto las inequidades económicas como la limitación en el ejercicio de sus derechos en salud. Todo esto es el resultado de un proceso histórico que ha fomentado la dependencia, la pérdida de identidad y la marginación de esta población. ${ }^{20,23}$ Los determinantes intermediarios (categorizados en circunstancias materiales-personales) son las condiciones de vida que se manifiestan en prácticas individuales y las características del ambiente, así como las condiciones de trabajo, la disponibilidad de alimentos, la salud, la educación y la vivienda; los logros no obtenidos subyacen por el crecimiento y distribución espacial de la población, para poder garantizar la satisfacción de las necesidades básicas. Los comportamientos biológicos se manifiestan a partir de la situación de salud de la población genética y los estilos de vida relacionados con las condiciones de riesgo para desarrollar algunas enfermedades, así como los hábitos alimenticios, que inciden sobre el estado nutricional de las personas. En el ámbito psicosocial implica a la comunidad que favorece o facilita el modo de vivir. ${ }^{23}$

En cuanto al sistema de salud, el acceso institucional permite incorporar los avances de la ciencia y tecnología al cuidado de la salud; ello modifica radicalmente al país. Así mismo las condiciones de vida de los individuos fortalecidas por políticas públicas y sociales de salud tienen como objetivo dar solución a las necesidades de los sectores más vulnerables de la población.

\section{CONCLUSIONES}

Los retos de organismos internacionales como la Organización Panamericana de la Salud (OPS) son la implementación de programas estratégicos que fomenten la participación ciudadana e involucren a la comunidad y sus organizaciones no gubernamentales en el cuidado de la salud. A partir de eso, a nivel central, en trabajos intersectoriales, se han desarrollado iniciativas centradas en la promoción a la salud que suponen la participación a la población.

En la historia de la salud en México, la política pública inédita, implícita en el sistema de protección social en salud, se estructura para asegurar que la población abierta cuente con servicios de salud acordes a su economía, con un acceso efectivo, oportuno y de calidad que satisfaga sus necesidades de salud. ${ }^{25}$ Para lograr una participación colaborativa, se involucra al personal de salud en la etapa preventiva, con diagnósticos oportunos y fomento a la salud en el primer nivel de atención. ${ }^{26}$

Los determinantes sociales en salud se atienden por medio de programas centrados en la prevención de las disparidades de salud en la población, con las siguientes premisas: repercutir directamente en la salud, predecir con mayor proporción la varianza del estado de salud (inequidad sanitaria), estructurar los comportamientos relacionados con la salud e interactuar mutuamente en la generación de la salud. ${ }^{23}$ Es importante explorar cuáles determinantes sociales y mecanismos involucrados en estos procesos conllevan a la aparición del síndrome metabólico y así prevenir los factores de riesgo, ya que ese padecimiento constituye en la actualidad un problema para la salud pública y tiene repercusión tanto en la salud física y psicológica como en el entorno social y económico.

\section{BIBLIOGRAFÍA}

1. OMS. WHO Obesity related publications. 2012. Disponible en: http:// www.who.int/nutrition/publications/obesity/en/index.html 
2. García GE et al. La obesidad y el síndrome metabólico como problema de salud pública. Revista de Salud Pública Méx. 2008; 50: (6).

3. PRONASA (Programa Nacional de Salud, 2011).

4. Zimmet P, Alberti G, Serrano M. Una nueva definición mundial del sín- drome metabólico propuesta por la Federación Internacional de Diabetes: fundamento y resultados. Rev Cardiología. 2005; 58 (12): 1371-1376.

5. Síndrome metabolico. Síndrome X. Disponible en: http://geosalud.com/ diabetesmellitus/smetabolico2.htm

6. Pérez R. Síndrome metabólico. Un desafío. Una epidemia. Una oportunidad. Rev Endocrinología y Nutrición. 2007.

7. Sánchez F, Jaramillo N, Vanegas A. Prevalencia y comportamiento de los factores de riesgo del síndrome metabólico según los diferentes intervalos de edad, en una población femenina del área de influencia de la Clínica Las Américas, en Medellín-Colombia. Rev Col Cardiol. 2008; 15 (3): 102-110.

8. González, E. M., Bautista, L., Irigoyen, A. Identificación de factores de riesgo para síndrome metabólico en población aparentemente sana de una unidad de medicina familiar en la ciudad de México Rev. Redalyc. 2009; 11(3): 127-135.

9. Mohan V, Mohan D. Síndrome metabólico en países en desarrollo. Diabetes Voice. 2006; (51)

10. Reyna MJ, Ziccardi A. Cohesión social y reducción de la pobreza. Las políticas y programas de cohesión social de la cooperación descentralizada. México; 2012.

11. Olaiz G, Rojas R, Aguilar C, Rauda J, Villalpando S. Diabetes mellitus en adultos mexicanos. Resultados de la Encuesta Nacional de Salud. Rev. Salud Pública. 2007; 49 (S.3).

12. Fernández BD et al. Síndrome metabólico en España: prevalencia y riesgo coronario asociado a la definición armonizada y a la propuesta por la OMS. Rev Esp Cardiol. 2012; 65 (3): 241-248.

13. González CA, Simental L, Elizondo AS, Sánchez ZJ, Gutiérrez SG, Guerrero RF. Prevalencia del síndrome metabólico entre adultos mexicanos no diabéticos, usando las definiciones de la OMS, NCEP-ATPIIIa e IDF. Rev. Médica del Hospital General. 2008; 71 (1): 11-19.
14. Manzur F, Alvear C, Alayon A. Caracterización fenotípica y metabólica del síndrome metabólico en Cartagena de Indias. Rev Col Cardiólogos. 2008; 15 (3): 97-101.

15. Schettini C, Schewdt E, Moreira V, Mogdasy C, Chávez L, Bianchi M et al. Prevalencia del síndrome metabólico en una población adulta. Rev Urug Cardiol. 2004; 19: 19-28.

16. González-López EM, Bautista SL, Irigoyen CA. Identificación de factores de riesgo para síndrome metabólico en población aparentemente sana de una unidad de medicina familiar en la ciudad de México. Rev Redalyc. 2009; 11 (3): 127-135.

17. AVISA, 2006. Años de Vida Saludable

18. INEGI 2008. Instituto Nacional de Estadística Geografía e Informática

19. Lalonde M. A new perspective on the health of the canadians. Ottawa: Minister of Supply and Services of Canada; 1974.

20. Agudelo S, Romero I. Concepto de determinantes de la salud y su estudio. 2010. Disponible en: http://www.slideshare.net/IvanRomero4/ determinantes-sociales-de-la-salud-7652021

21. Breilh P, Miño J. Epidemiología entrefuegos. Medellín: Memorias de la Asociación Latinoamericana de Medicina Social; 1994.

22. Castellanos PL. "Sobre el concepto de salud-enfermedad. Un punto de vista epidemiológico”. Caracas, Venezuela: Cuadernos Médico-Sociales; 1987.

23. Comisión de Determinantes Sociales (CDSS-2008), OMS (2010). Comisión sobre Determinantes Sociales en Salud; 2005-2008.

24. Breilh P, Miño J. Los determinantes sociales versus determinación social Brasil; 2012.

25. González-Robledo LM, Nigenda G, González-Robledo MC, Reich M. Separación de funciones en el sistema de protección social en salud. Salud Pública Méx. 2011; 53 (4): 416-424.

26. Granados-Cosme JA, Tetelboin HC, Torres CC, Pineda PD, VillaContreras BM. Operación del programa del Sistema Integral de Calidad en Salud (SICALIDAD). La función de los gestores en atención primaria y hospitales. México: Salud Pública. 2011; 4: 402-406.

27. OMS. Atención primaria de salud informe de la comisión internacional sobre atención primaria de salud. Alma-Ata URSS. Ginebra: OMS; 1978. pp. 6-12. 